\title{
INFECTIOUS DISEASES, NSW: JUNE 2000
}

\section{TRENDS}

Notifications of infectious diseases to the end of April 2000 were in line with seasonal expectations (Figure 7, Table 3). Notably, the late summer peak in arbovirus disease notifications (largely due to 247 Ross River virus infections, and 48 Barmah Forest virus infections in the three-month period to the end of April) was smaller than in most recent years.

The expected autumn peak in legionnaires disease has appeared with eight cases in NSW reported in April. No causal link has been identified for these cases, although cases are thought to increase in NSW as air-conditioning systems that use cooling towers are operated inconsistently as the weather cools down. This inconsistent use could result in an increased creation of aerosols (aerosolisation), allowing subsequent inhalation of any resident legionella bacteria. Building operators must ensure that cooling towers are properly cleaned and disinfected to minimise the risk that they will harbour these bacteria.

In April, the Victorian Department of Human Services reported a large outbreak of Legionnaires disease cased by Legionella pneumophila infections linked to visiting the recently opened Melbourne Aquarium. As of 11th May, 91 confirmed cases associated with the outbreak had been reported, including two deaths. Cases were aged between 23-89 years old, and reported visiting the Aquarium between 11-25 April.

The urinary antigen test for L. pneumophila serogroup 1 has proven useful in establishing the diagnosis in many recent cases. Clinicians are encouraged to use this test in the management of suspected cases.

Cases of meningococcal disease can be expected to increase with the onset of winter. Clinicians are reminded of the importance of early treatment with intravenous antibiotics for suspected cases, and notification of such cases to the local Public Health Unit. The Public Health Unit can then assist in the identification of contacts at risk, and institute preventive measures. The Public Health Unit can also advise on newly available diagnostic tests, including PCR and serological assays.

\section{INTRODUCTION OFTHE AUSTRALIAN STANDARD VACCINATION SCHEDULE}

The National Health and Medical Research Council (NHMRC) has endorsed the Australian Standard Vaccination Schedule (ASVS) that commenced on 1st May 2000 (Table 2), which heralds the introduction of universal infant hepatitis B vaccination. Babies born prior to that date will remain on the previous NHMRC immunisation schedule.

The ASVS offers a choice of two 'paths' to incorporate two new combination vaccines. NSW Health has chosen to follow Path 1 of the ASVS, which will introduce the new combination vaccine, InfanrixHepB.

To ensure the smooth implementation of the new ASVS, all general practitioners will receive supporting information, including the new NHMRC Australian Immunisation Handbook, seventh edition, from the Commonwealth in May 2000. Additional copies may be obtained by phoning 1800671811 . Hospitals, Community Health Centres and other service providers will receive their copies of the handbook from their local Public Health Unit.

Copies of the NSW Immunisation Schedule, a new vaccine order form, and a hepatitis B pamphlet have been widely distributed to service providers. The NSW Immunisation Schedule will also be available on the NSW Department of Health Web site. 


\section{TABLE 2}

\section{NSW IMMUNISATION SCHEDULE 2000}

Babies born BEFORE 1 May Babies born on or AFTER 1 May

\begin{tabular}{|c|c|c|c|c|c|}
\hline AGE & ANTIGEN & VACCINE & AGE & ANTIGEN & VACCINE \\
\hline 2 months & $\begin{array}{l}\text { DTPa } \\
\text { Hib } \\
\text { Polio }\end{array}$ & $\begin{array}{l}\text { Infanrix } \\
\text { HibTITER/PedvaxHIB* } \\
\text { OPV }\end{array}$ & $\begin{array}{l}\text { Birth } \\
\text { (maternity units) }\end{array}$ & Hep B & H-B-Vax II \\
\hline 4 months & $\begin{array}{l}\text { DTPa } \\
\mathrm{Hib}\end{array}$ & $\begin{array}{l}\text { Infanrix } \\
\text { HibTITER/PedvaxHIB* }\end{array}$ & 2 months & $\begin{array}{l}\text { DTPa-HepB } \\
\text { Hib }\end{array}$ & $\begin{array}{l}\text { InfanrixHepB } \\
\text { PedvaxHIB }\end{array}$ \\
\hline & Polio & OPV & & & Polio OPV \\
\hline 6 months & $\begin{array}{l}\text { DTPa } \\
\text { Hib } \\
\text { Polio }\end{array}$ & $\begin{array}{l}\text { Infanrix } \\
\text { HibTITER } \\
\text { OPV }\end{array}$ & 4 months & $\begin{array}{l}\text { DTPa-HepB } \\
\text { Hib } \\
\text { Polio }\end{array}$ & $\begin{array}{l}\text { InfanrixHepB } \\
\text { PedvaxHIB } \\
\text { OPV }\end{array}$ \\
\hline 12 months & $\begin{array}{l}\text { MMR } \\
\mathrm{Hib}\end{array}$ & $\begin{array}{l}\text { Priorix } \\
\text { PedvaxHIB* }\end{array}$ & 6 months & $\begin{array}{l}\text { DTPa-Hep B } \\
\text { Polio }\end{array}$ & $\begin{array}{l}\text { InfanrixHepB } \\
\text { OPV }\end{array}$ \\
\hline 18 months & $\begin{array}{l}\text { DTPa } \\
\mathrm{Hib}\end{array}$ & $\begin{array}{l}\text { Infanrix } \\
\text { HibTITER }\end{array}$ & 12 months & $\begin{array}{l}\text { MMR } \\
\mathrm{Hib}\end{array}$ & $\begin{array}{l}\text { Priorix } \\
\text { PedvaxHIB }\end{array}$ \\
\hline 4 years & $\begin{array}{l}\text { DTPa } \\
\text { MMR } \\
\text { Polio }\end{array}$ & $\begin{array}{l}\text { Infanrix } \\
\text { Priorix } \\
\text { OPV }\end{array}$ & 18 months & $\mathrm{DTPa}$ & Infanrix \\
\hline $\begin{array}{l}10 \text { years } \\
1 \text { mths later } \\
5 \text { mths later }\end{array}$ & $\begin{array}{l}\text { Hep B (1) } \\
\text { Hep B (2) } \\
\text { Hep B (3) }\end{array}$ & H-B-Vax II & 4 years & $\begin{array}{l}\text { DTPa } \\
\text { MMR } \\
\text { Polio }\end{array}$ & $\begin{array}{l}\text { Infanrix } \\
\text { Priorix } \\
\text { OPV }\end{array}$ \\
\hline $15-19$ years & $\begin{array}{l}\text { ADT } \\
\text { Polio }\end{array}$ & $\begin{array}{l}\text { ADT } \\
\text { OPV }\end{array}$ & 15-19 years & $\begin{array}{l}\text { ADT } \\
\text { Polio }\end{array}$ & $\begin{array}{l}\text { ADT } \\
\text { OPV }\end{array}$ \\
\hline
\end{tabular}

* Aboriginal \& Torres Strait Islander children born before the 1st of May 2000 should continue to receive PedvaxHIB. All children born on or after that date should receive PedvaxHIB. 


\section{FIGURE 7}

REPORTS OF SELECTED INFECTIOUS DISEASES, NSW, JANUARY 1995 TO APRIL 2000, BY MONTH OF ONSET

These are preliminary data: case counts in recent months may increase because of reporting delays

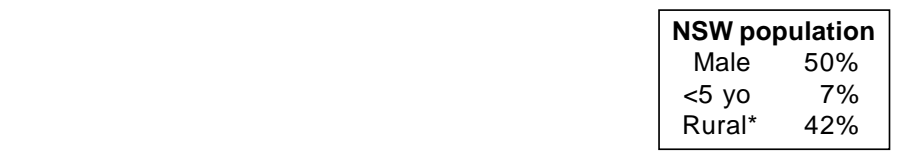

\begin{tabular}{|cc|}
\hline \multicolumn{2}{|c|}{ Feb $00-A p r ~ 00$} \\
Male & $54 \%$ \\
$<5$ yo & $0 \%$ \\
Rural & $92 \%$ \\
\hline
\end{tabular}

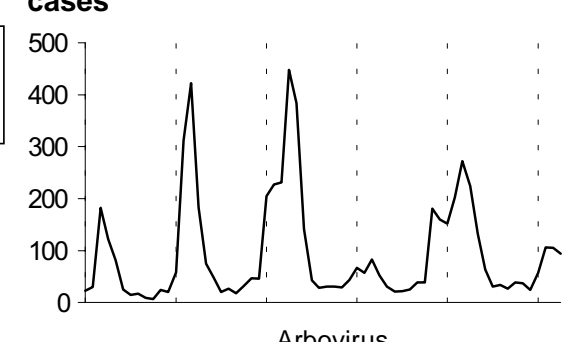

cases
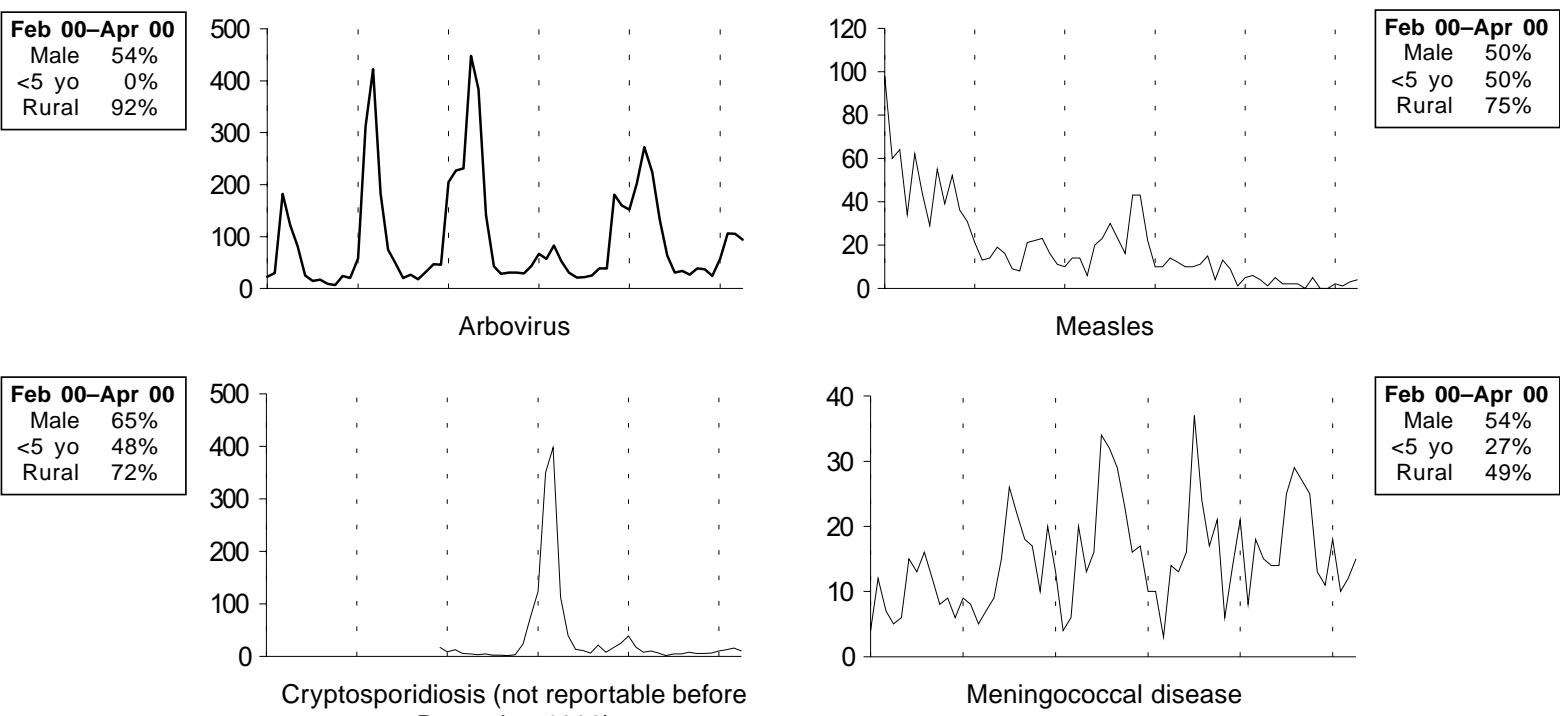

Feb 00-Apr 00

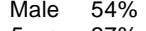

$<5$ yo $27 \%$

Rural $72 \%$

\section{December 1996)}

Meningococcal disease

\begin{tabular}{|c|c|}
\hline \multicolumn{2}{|c|}{ Feb $00-A p r 00$} \\
\hline Male & $90 \%$ \\
\hline$<5$ yo & $<1 \%$ \\
\hline Rural & $18 \%$ \\
\hline
\end{tabular}
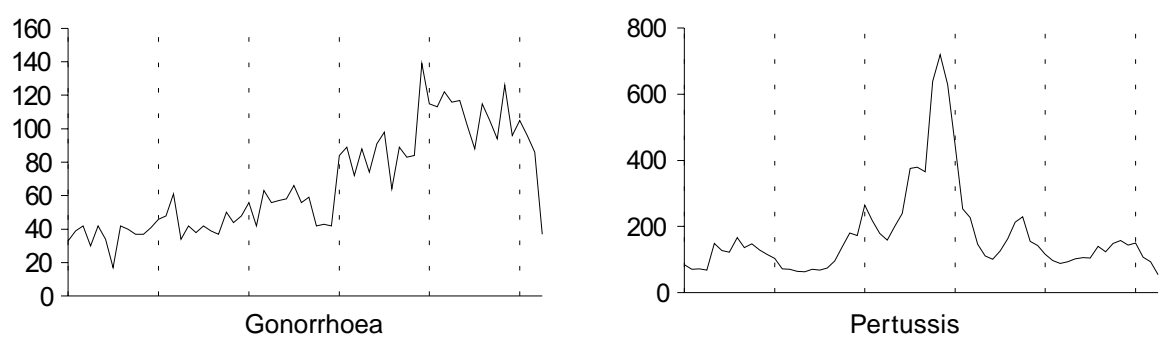

Feb 00-Apr 00

Male $44 \%$

$<5$ yo $12 \%$

Rural $61 \%$

Gonorrhoea

Pertussis
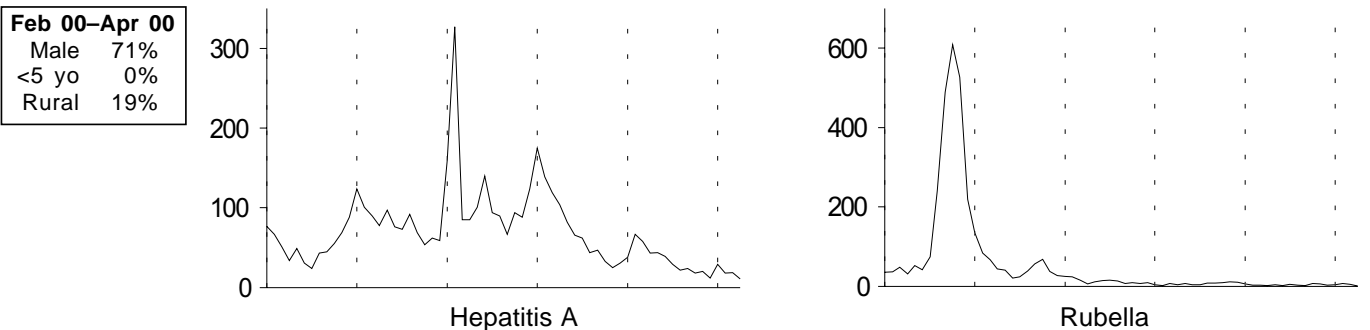

Feb 00-Apr 00

Male $85 \%$

$<5$ yo $8 \%$

Rural $31 \%$

Rural 19\%

Hepatitis A
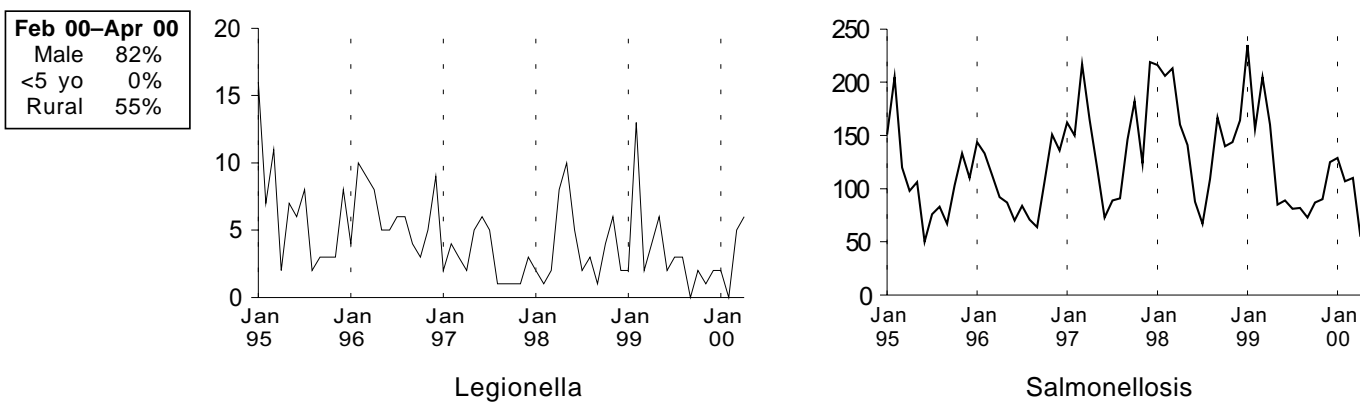

* For definition, see NSW Public Health Bulletin, April 2000 


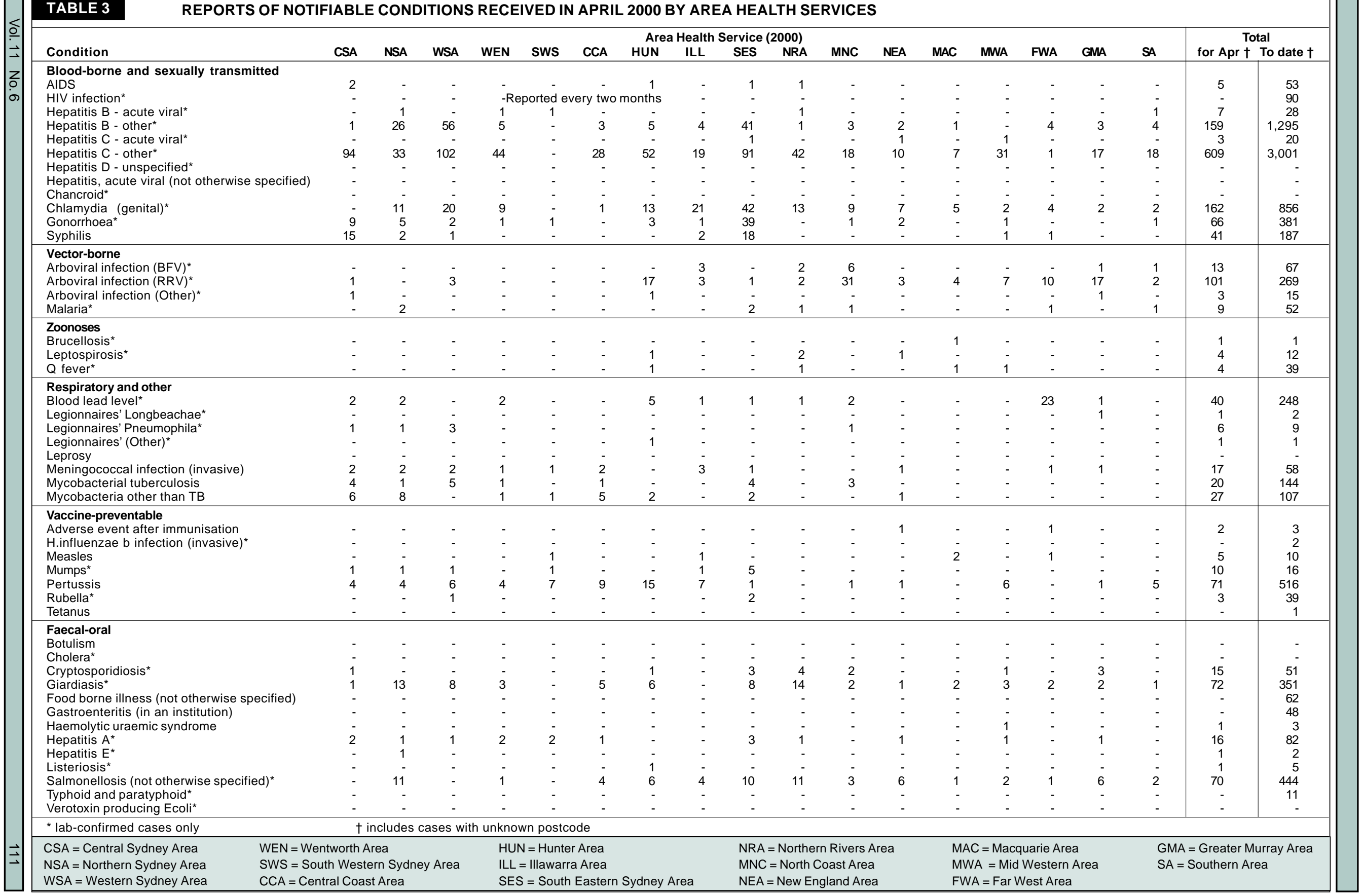

